\title{
ESTUDIOS
}

\section{Vicente Bianchi Alarcón, una biografía musical y las implicancias de haber obtenido el Premio Nacional de Artes Musicales 2016}

Vicente Bianchi Alarcón, a musical biography, and the implications of having won the National Prize of Musical Arts in 2016

\author{
por \\ Álvaro Menanteau Aravena \\ Universidad de Santiago de Chile, Chile \\ alvaro.menanteau@usach.cl
}

El presente artículo consta de dos secciones. En la primera parte se revisa la trayectoria musical de Vicente Bianchi Alarcón, en sus variadas dimensiones como pianista, arreglador, director orquestal y compositor. La mayor parte de la información contenida fue obtenida de conversaciones con Vicente Bianchi, en particular de una entrevista realizada en 2002. La segunda parte contiene una relación de datos y consideraciones respecto de la obtención del Premio Nacional de Artes Musicales 2016.

Palabras clave: Vicente Bianchi, Chile, música popular, Premio Nacional de Artes Musicales.

This article consists of two sections. In the first part we review the musical trajectory of Vicente Bianchi Alarcon, in his varied dimensions as a pianist, arranger, orchestral conductor and composer. Most of the information contained was obtained from conversations with Vicente Bianchi, especially from an interview made in 2002. The second part contains a list of data and considerations regarding the obtaining of the National Prize for Musical Arts 2016.

Keywords: Vicente Bianchi, Chile, popular music, National Prize for Musical Arts

\section{VICENTE BIANCHI, UNA BIOGRAFÍA MUSICAL}

\subsection{Sus inicios musicales}

Francisco Vicente Germán Bianchi Alarcón nació el 27 de enero de 1920 en Santiago de Chile, en la comuna de Ñuñoa. Fue el mayor de los cinco hijos del matrimonio de Germán Bianchi y Blanca Alarcón. Luego de Vicente nacieron sus hermanas Elizabeth, Raquel, Blanca, y su hermano menor, Claudio. Su padre era constructor y su madre dueña de casa. Ella tocaba un poco de piano. Desde 
muy niño a Vicente le gustaba escuchar los discos en la vitrola ${ }^{1}$ que había en casa, junto al piano. Debido a estos intereses, a los seis años sus padres le propusieron que estudiara música, consiguiendo como profesor a un pianista profesional que tocaba en las funciones de cine mudo. Este primer profesor, Daniel Julio, era vecino y amigo de su familia, quien cada día, luego de sus labores profesionales, le hacía clases en las noches. Bianchi avanzó rápido con las lecciones, pero debido a los compromisos laborales de Julio, tuvo que cambiar de profesor. Entonces estudió tres años con Olga Águila Fraga, para luego ingresar al Conservatorio Nacional de Música, cuando ya había cumplido los 10 años de edad.

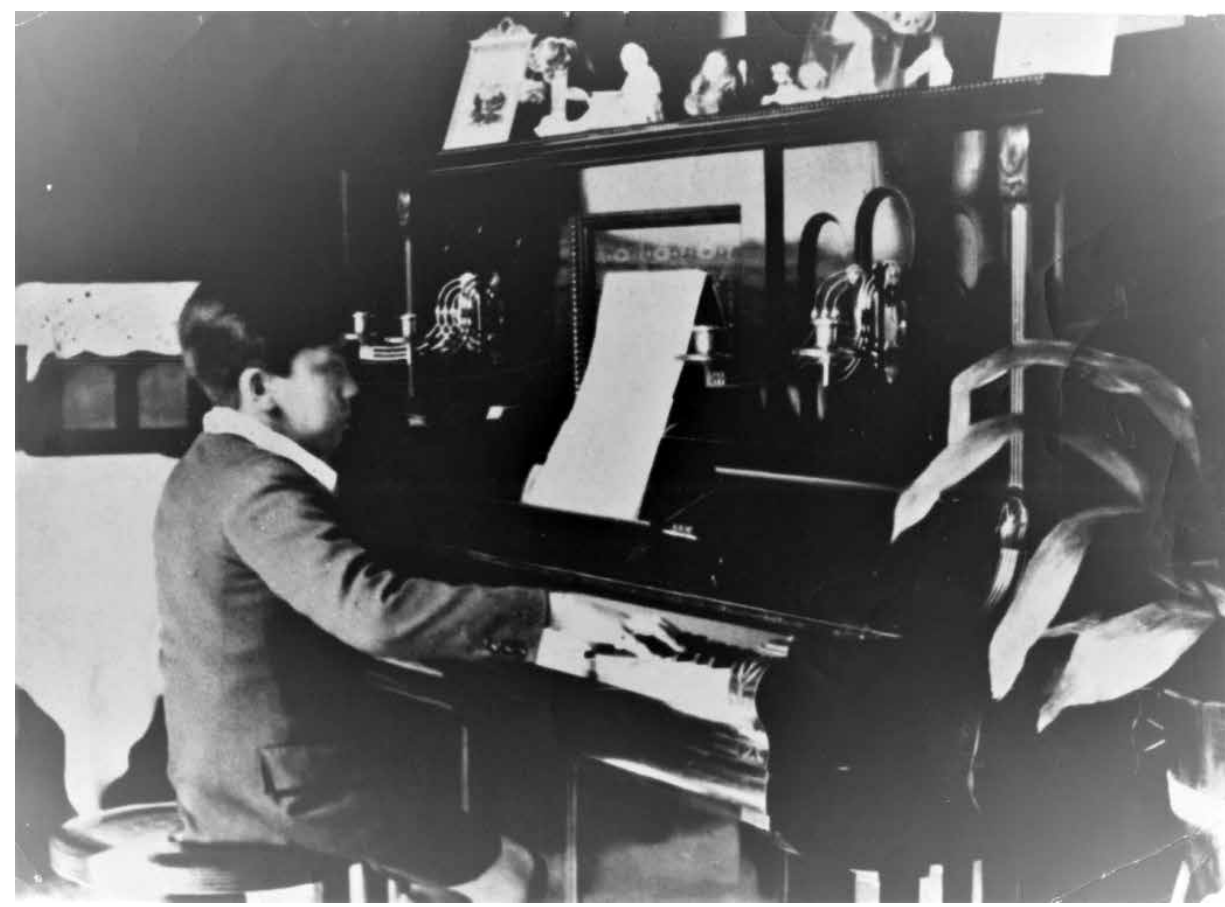

Foto 1. Vicente Bianchi tocando piano a los 9 años. 1929.

En el Conservatorio Nacional de Música estudió durante seis años, período en el que fue alumno de Flora $\mathrm{Vial}^{2}$ en teoría musical, armonía con Samuel Negrete, y piano con Alberto Spikin-Howard. La experiencia de haber estudiado música de manera sistemática, habilitó a Bianchi para combinar aquellos conocimientos técnicos con la práctica de la música popular en el medio radiofónico. Esto debido a que, simultáneamente a sus estudios en el Conservatorio Nacional de Música,

\footnotetext{
1 Especie de tocadiscos, llamado así por haber sido patentada por el sello RCA Víctor (N. del E.)

2 Flora Vial fue la madre de la pianista Flora Guerra Vial (1918-1993).
} 
Bianchi integró la orquesta infantil del programa radial El abuelito Luis, que se transmitía por radio Otto Becker. Se trataba de un conjunto instrumental muy particular, con varios cantantes, piano, acordeón, violín y batería. Como parte del elenco de este programa radial, Bianchi realizó al menos dos giras por el país. Hasta los 17 años trabajó con la orquesta de aquel programa, en donde tuvo contacto con futuros músicos profesionales como el chelista y futuro director Juan Mateucci, el cantante Humberto Lozán y el pianista Fernando Morello ${ }^{3}$.

A los 17 años abandonó el trabajo en radio Otto Becker, cuando en radio La Americana le ofrecieron un trabajo con contrato exclusivo. Allí Bianchi fue testigo de los inicios musicales de Los Quincheros ${ }^{4}$. Esta nueva situación laboral, sumada al ambiente que vivía en el Conservatorio, llevó a Vicente Bianchi a tomar la decisión de abandonar sus estudios formales ${ }^{5}$, para dedicarse completamente al trabajo en el ambiente radiofónico de la época.

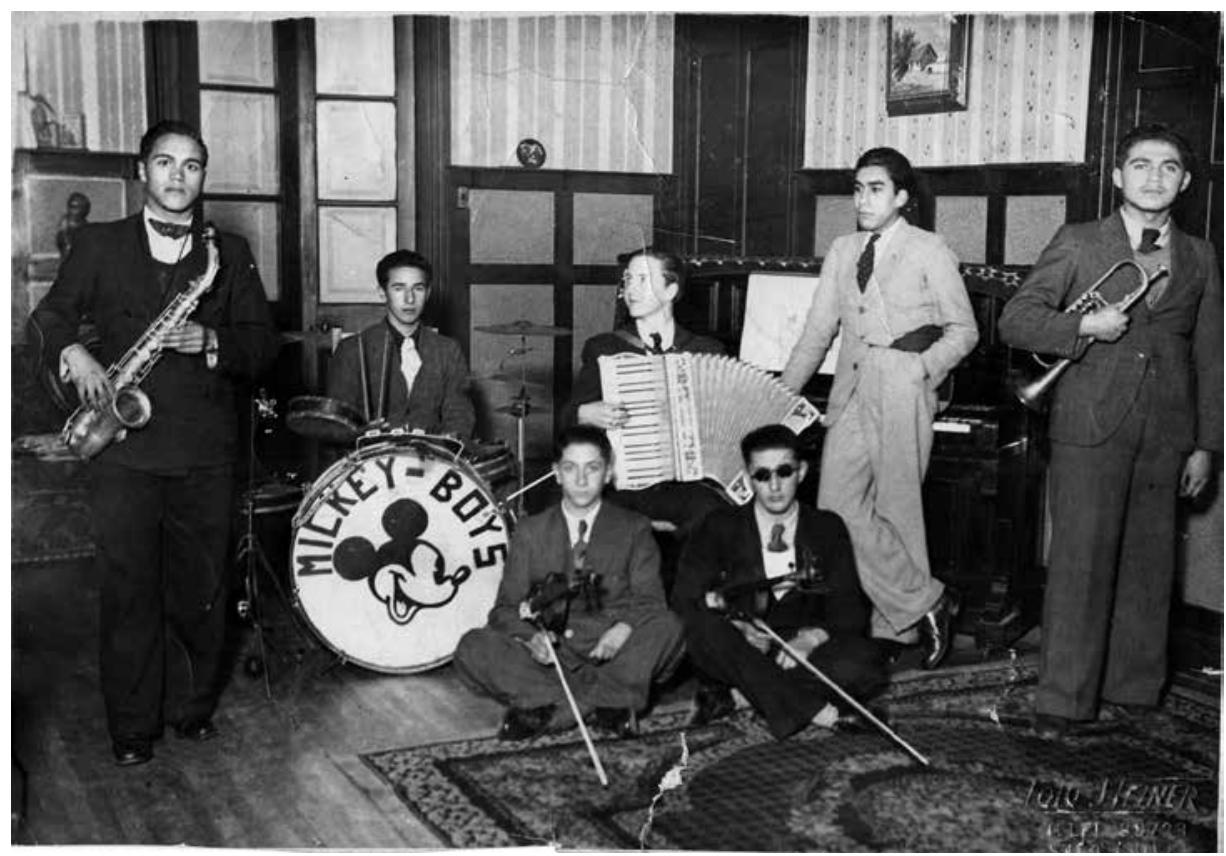

Foto 2. Bianchi (de pie sobre el piano) en uno de sus conjuntos de jazz, con Fernando Morello en batería, 1938.

3 Morello también fue baterista en un grupo de música popular que formó Vicente Bianchi a los 18 años; junto a Lozán, Fernando Morello formó parte de la primera alineación de la Orquesta Huambaly. En la orquesta de niños de El abuelito Luis también participó como cantante Inés Venegas, madre de los jazzistas Roberto, Mario y Pablo Lecaros.

4 Conjunto vocal que pasó a llamarse Los Huasos Quincheros después de 1957.

5 Bianchi reconoce que le incomodaba el exceso de celo de los profesores, quienes criticaban la actividad paralela de sus alumnos en música popular. Otra situación que le incomodaba era la fuerte presión por emular el modelo representado por Claudio Arrau. 
En el contexto de los músicos más jóvenes de su generación, Vicente Bianchi tuvo la ventaja de poder aplicar los conocimientos adquiridos en el Conservatorio a la actividad de música popular. Una de sus primeras experiencias formando su propio grupo musical, fue una banda de jazz que integraba junto con Luis Silva en guitarra y Dolores Díaz en canto ${ }^{6}$ con scat. Sin embargo, estas experiencias circunstanciales fueron luego remplazadas por el trabajo profesional en radio.

\subsection{Su actividad en radios y sellos discográficos}

Su primer trabajo en radio La Americana implicaba poner al aire números musicales desde el piano, ya sea en cortinas musicales o acompañando cantantes. Esta actividad correspondía a lo que en aquel tiempo se denominaba fantasista. En el caso de los acompañamientos, esto se hacía leyendo arreglos musicales, improvisando, o bien transportando la música a las tonalidades adecuadas para cada cantante.

En 1940 fue contratado en radio Agricultura, una emisora más grande y con mayores recursos, donde pudo trabajar con una orquesta estable y comenzar a escribir sus propios arreglos. En radio Agricultura le correspondió acompañar al tenor lírico italiano Tito Schipa ${ }^{7}$, con quien se reencontró años después en Lima.

La primera salida profesional de Bianchi fuera de Chile aconteció en 1943, contratado por radio El Mundo, de Buenos Aires. En aquella época esa radio era considerada la emisora con la señal más poderosa en América Latina, siendo dirigida por Jorge Aguirre, hijo del compositor argentino Julián Aguirre. Allí Bianchi estuvo trabajando seis meses, en programas que demandaban tocar al piano toda clase de melodías de moda, ya sea creando sus propios arreglos o improvisando.

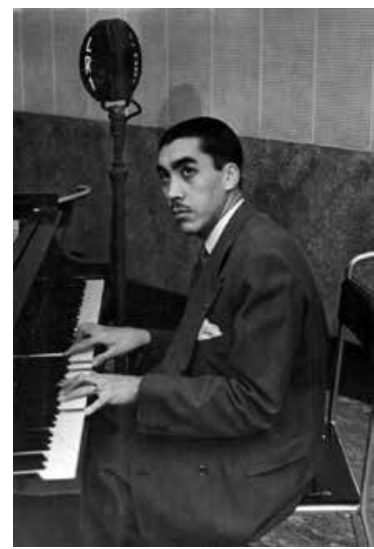

Foto 3. En Buenos Aires, contratado por radio El Mundo. 1943.

${ }^{6}$ Luis Silva fue un pionero del jazz en Chile, cultor del estilo de jazz gitano impuesto en Europa por Django Reinhardt; posteriormente Silva integró el dúo folclórico Los Perlas. Por su parte, Dolores Díaz (sobrina de Eloísa Díaz, primera médico titulada en Chile y Sudamérica) actuaba con el nombre artístico Dolly Day.

7 Otras visitas internacionales con las que Bianchi pudo compartir en esos primeros años de actividad profesional fueron la bailarina Josephine Baker y la actriz Marlene Dietrich. 
A su regreso de Buenos Aires, Bianchi ingresó a trabajar en radio Minería. Entre los cantantes populares que acompañó con su orquesta estuvo el bolerista Lucho Gatica, con quien realizó además importantes grabaciones, haciéndose cargo de los arreglos musicales y la dirección orquestal. En radio Minería pudo disponer de una orquesta más grande, que podía aumentar hasta 30 músicos, de acuerdo con las necesidades de los programas. En este contexto de trabajo, Bianchi comenzó a escribir regularmente arreglos orquestales de música tradicional chilena. De ahí surgió además un conjunto para interpretar canciones folclóricas con las hermanas Estela y Margot Loyola.

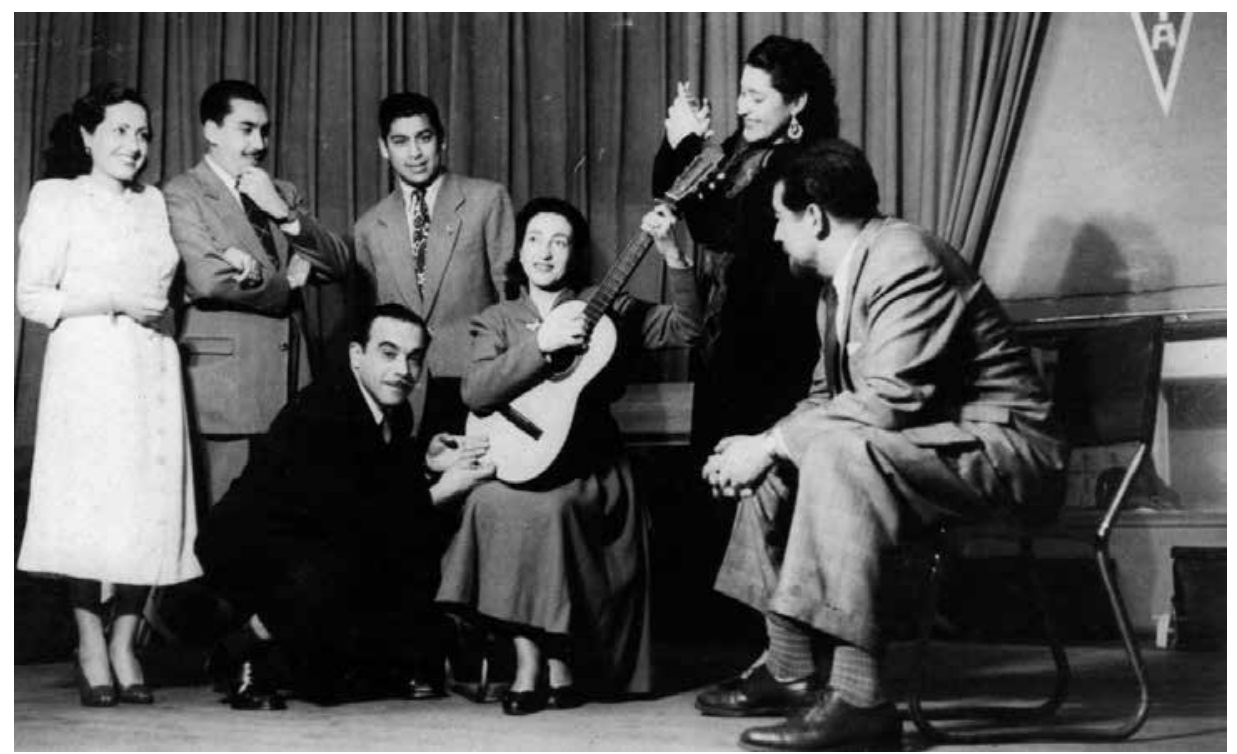

Foto 4. En radio Minería junto con las Hermanitas Loyola y la cantante Magda. c.1948.

En radio Minería también tuvo la posibilidad de montar pequeños conciertos de música clásica, aprovechando la presencia de Enrique Iniesta ${ }^{8}$ como solista en violín. Se debe recordar que por aquellos años un importante número de músicos de cuerda y vientos de la Orquesta Sinfónica de Chile trabajaban en las radios, participando en los programas que se difundían por ese medio. Con Iniesta como solista, Bianchi pudo estrenar obras orquestales de Saint-Saëns y de Enrique Soro, pianista y compositor académico con quien mantuvo amistad durante muchos años.

De esta etapa de actividad en radios, Bianchi recuerda con especial afecto su trabajo con violinistas y otros intérpretes de cuerda que pertenecían al circuito de

8 Destacado violinista de origen español, radicado por 20 años en Chile, donde fue concertino de la Orquesta Sinfónica de Chile y profesor en el Conservatorio Nacional de Música. 
la música clásica ${ }^{9}$. Por ejemplo, su arreglo de la tonada La tranquera, de Osmán Pérez Freire, fue grabada por Bianchi en 1958 junto a César Araya en violín, Agustín Cullel en viola, Arnaldo Fuentes en chelo y Ramón Bignon en contrabajo. Con todos ellos había trabajado en la radio y en sesiones de grabación para los sellos discográficos de la época.

En paralelo con su actividad radial, Vicente Bianchi obtuvo su primer contrato discográfico con RCA Victor, sello para el que grabó varios discos acompañando a las hermanas Sonia y Myriam, y al cantante Mario Arancibia.

En 1950 Bianchi fue contratado para realizar algunas presentaciones en radio El Sol, de Lima. Se trataba de un trabajo puntual, que no debía durar más de un mes, pero Bianchi terminó quedándose en Perú por cuatro años. El contrato que lo llevó a Lima consistía en presentaciones en la recién inaugurada radio El Sol, acompañando en el piano al Trío Llanquiray, grupo vocal que había formado en Chile $^{10}$. Dicho trío estaba compuesto por las hermanas Murúa, entre ellas se hallaba Hely Murúa, esposa de Bianchi desde 1947. Con este grupo se presentaron en radio El Sol y en el teatro Bolívar de Lima. Tuvieron un éxito rotundo, lo que se tradujo en la prolongación de su contrato, y luego la oferta de un contrato indefinido como director musical de la radio.

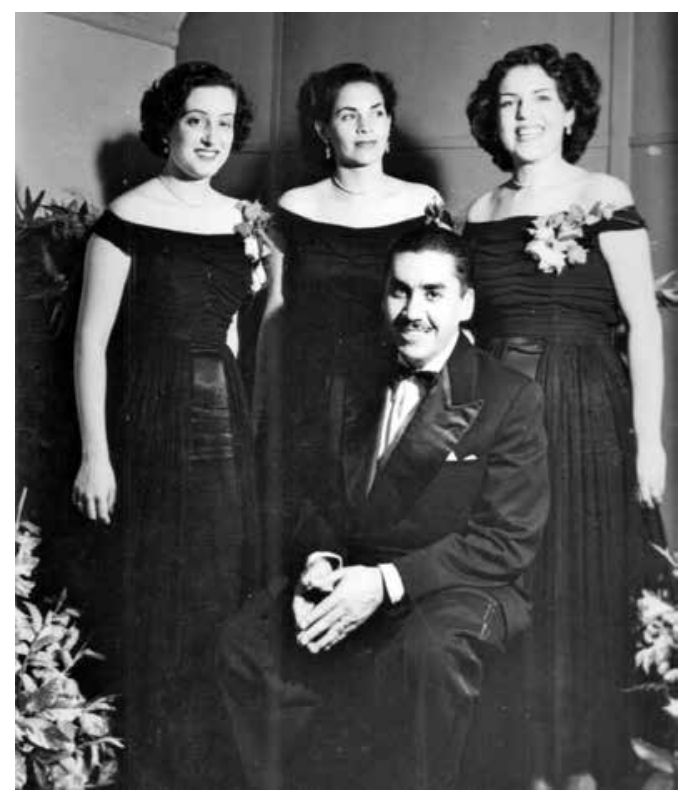

Foto 5. Junto al Trío Llanquiray. Lima, 1950.

9 Otros violinistas que integraron las orquestas que organizaba Bianchi fueron Stefan Tertz, Alberto Dourthé, Pedro d'Andurain y Jaime de la Jara.

10 A su regreso a Chile, el Trío Llanquiray se transformó en el conjunto Los Jaranistas, con el que Bianchi siguió cultivando el cancionero popular peruano. 
En radio El Sol tuvo la posibilidad de dirigir una orquesta de al menos 16 músicos, que en ocasiones podía aumentar a 30. En aquellas circunstancias Bianchi comenzó a hacer arreglos de músicas populares y folclóricas de Perú, destacándose sus arreglos de valses, marineras y tonderos, junto con repertorios de música andina y afroperuana ${ }^{11}$. Esos arreglos los presentaba en los programas orquestales o en los intermedios que se requerían dentro de la programación radial. Aunque la industria fonográfica no estaba tan desarrollada en Perú, Bianchi también tuvo la posibilidad de grabar algunos ejemplos de sus arreglos orquestales para el sello RCA Victor en Lima. Junto con lo anterior, también tuvo la posibilidad de componer algunas canciones populares, como el vals Peruanita bonita ${ }^{12}$.

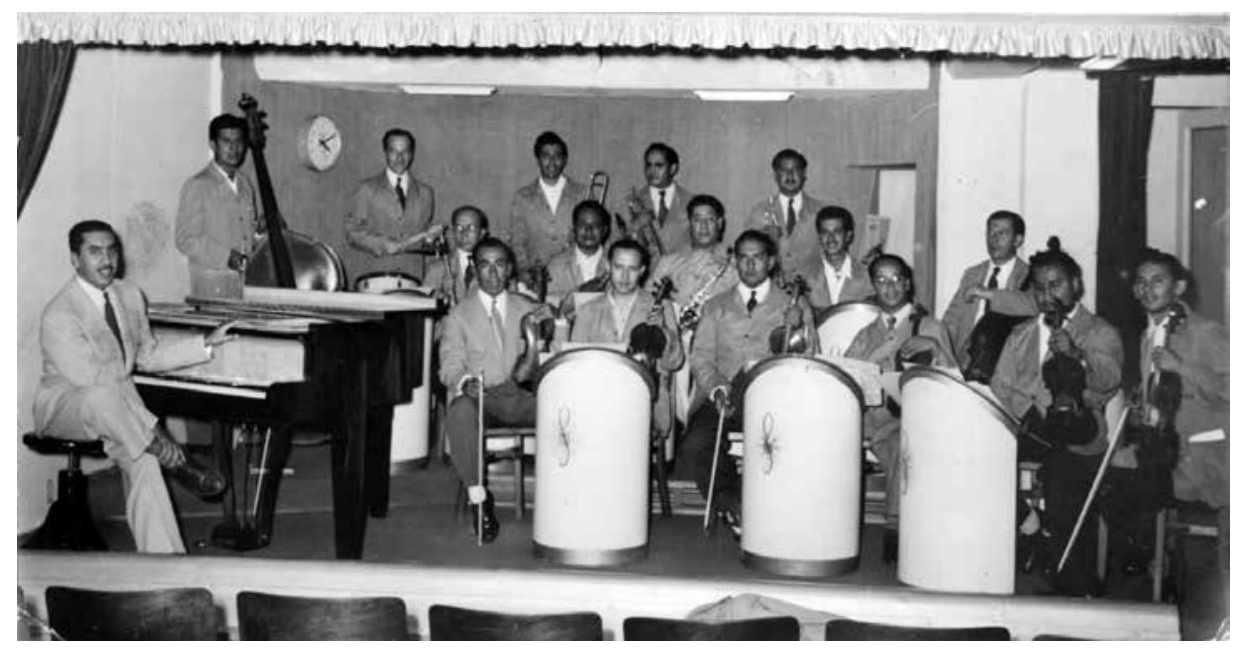

Foto 6. Vicente Bianchi con la orquesta de radio El Sol. Lima, 1950.

Vicente Bianchi regresó a Chile en enero de 1955, siendo de inmediato contratado por el sello Odeón, gracias a un contacto previo que había hecho en Lima con el cantante Lucho Gatica. Como artista exclusivo del sello Odeón, Bianchi debió grabar acompañando a otros músicos del sello. Fue así como realizó gran cantidad de grabaciones de boleros y canciones populares con Lucho Gatica, Sonia y Myriam, y Los Huasos Quincheros, quienes también habían dejado el sello RCA Victor.

11 Consultar Catálogo selectivo de las obras musicales de Vicente Bianchi Alarcón publicado en este número, O-15, O-16, O-19 a O-25.

12 Catálogo, O-31. 


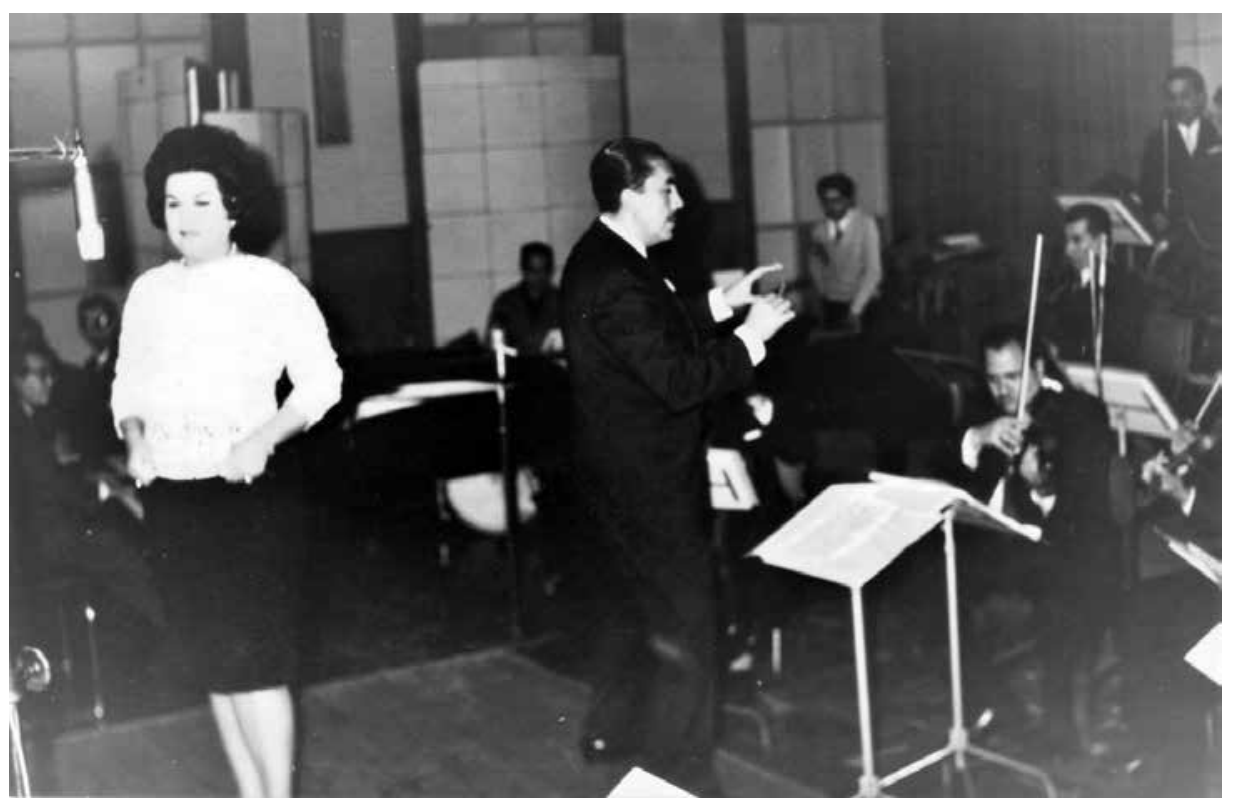

Foto 7. Vicente Bianchi acompañando a Ester Soré en radio Minería. c.1958

Paralelamente se reinsertó al medio radiofónico chileno mediante un contrato con radio Cooperativa, la última radioemisora en la que trabajó, hasta fines de la década de 1960. Entre los exponentes de música popular que acompañó allí se contaba a Arturo Gatica, Hilda Sour, Silvia Infantas, Pedro Vargas, Leo Marini y la cantante lírica Rayén Quitral. Fue en los estudios de radio Cooperativa en que se hicieron los primeros ensayos y presentaciones de su Misa a la Chilena, ante las autoridades eclesiásticas locales.

\section{VICENTE BIANCHI Y LA COMPOSICIÓN MUSICAL}

Como ha quedado establecido previamente, la formación musical de Vicente Bianchi en el Conservatorio Nacional de Música lo habilitó no solo como pianista, sino además como arreglador musical. Estos aspectos fueron centrales para su desempeño como músico popular vinculado a la actividad profesional en radios y sellos grabadores. Si Bianchi era disputado por radioemisoras y sellos discográficos, ello se debía a su dominio de la teoría musical y su desempeño como pianista y director orquestal. El perfil del director orquestal de música popular de aquella época exigía un alto desempeño en la dirección, la escritura de arreglos y el acompañamiento al piano. Otros músicos con un perfil profesional similar a Vicente Bianchi eran Pedro Mesías, Rafael Hermosilla y Federico Ojeda, quienes también dominaban estas tres áreas, pero Bianchi agregaba a estas habilidades sus capacidades como compositor. 
Su primera composición propiamente tal la realizó a los 12 años. Preludio melancólico ${ }^{13}$, inspirado en el estilo pianístico de Chopin, solo fue grabado en 2010, y publicado al año siguiente ${ }^{14}$. Pero el grueso de la producción musical de Bianchi como compositor se sitúa en el ámbito de la canción popular.

La primera composición de éxito masivo fue la canción Amanecer ${ }^{15}$, que era parte de la banda sonora de la película Amanecer de esperanzas (1941) de Miguel Frank. Esta canción fue muy popular en su tiempo, siendo uno de los números habituales en los concursos de aficionados que realizaban las radios de la época. Además, una versión orquestal de Amanecer fue utilizada en los inicios de las funciones del cine Rex, de Santiago ${ }^{16}$. De ahí en adelante surgieron otras canciones populares, como los valses Rayito de sol ${ }^{17}$ y Peruanita bonita, el bolero No te vayas amor ${ }^{18}$, el cuando Consejos por casamiento ${ }^{19}$, la refalosa La refalaita ${ }^{20}$, y las tonadas Chile compañero y La independencia de Chile ${ }^{21}$. En el contexto de las composiciones instrumentales (en versiones tanto orquestales como para piano solo) se destacan el one step Abejorros ${ }^{22}$, y las piezas de fantasía Scherzando y Tonada rítmica ${ }^{23}$. Entre sus obras religiosas de mayor formato sobresalen su Misa a la Chilena (1964), la Misa de la Cruz del Sur (1969) y el Te Deum laudamus (1969)24.

Junto con las obras mencionadas, ocupan un lugar relevante sus composiciones basadas en poemas de Pablo Neruda. La asociación creativa entre Bianchi y Neruda no solo representó un momento culminante en la trayectoria profesional del compositor, sino además un instante destacado en el cruce entre la poesía, la música tradicional y la industria musical chilenas. Mientras estaba trabajando en Lima, Bianchi recibió unos textos de Neruda que le había enviado un amigo desde Chile. Bianchi reconoce que intentó musicalizarlos estando en Lima, pero sin resultado. A su regreso a Chile en 1955, compuso las Tonadas de Manuel Rodríguez, pieza que Bianchi presentó a Neruda y obtuvo su permiso para seguir musicalizando otros poemas.

13 Catálogo, O-1.

14 Ver Menanteau 2011.

15 Catálogo, O-4.

16 Ver Ponce en http://www.musicapopular.cl/artista/vicente-bianchi/

17 Catálogo, O-5.

18 Bolero que le presentara Bianchi a Lucho Gatica cuando se encontraron en Lima en 1954, y que luego grabaron en Chile. Catálogo, O-26.

19 Grabado por Silvia Infantas y Los Baqueanos para el sello Odeón, junto con la Orquesta de Vicente Bianchi. Catálogo, O-40.

20 Catálogo, O-43.

21 Catálogo, O-28 y O-120, respectivamente.

22 Catálogo, O-2.

23 Catálogo, O-3 y O-7, respectivamente.

24 Catálogo, O-67 y O-76 y O-75, respectivamente. 
Ese mismo año fueron grabadas las Tonadas de Manuel Rodríguez, Canto a Bernardo O'Higgins y Romance de los Carrera ${ }^{25}$ en un disco LP por Silvia Infantas y Los Baqueanos, bajo el título de Música para la historia de Chile. Aquel disco tuvo una destacada difusión masiva, tanto por la venta física del fonograma como por las presentaciones de este repertorio en radios y escenarios ${ }^{26}$.

En 1998 su canción La noche de Chillán ${ }^{27}$ obtuvo el primer lugar en la competencia folclórica del Festival de la Canción de Viña del Mar. Esta canción fue producto de la musicalización de un poema de Pablo Neruda, quien había entregado personalmente a Bianchi el manuscrito en 1973.

\section{ADAPTÁNDOSE A LOS CAMBIOS}

En la década de 1960 la actividad radial comenzaba a decaer. La costosa producción musical que implicaba la manutención de auditorios radiales y de una orquesta en vivo, comenzó a ser desplazada por la televisión y el impacto de la música popular estadounidense, en particular del rock and rolly su influencia en la naciente cultura juvenil. Todo esto trajo importantes cambios en la industria musical chilena: los conjuntos se electrificaron, disminuyeron de tamaño y los repertorios de origen anglosajón desplazaron a los estilos cultivados anteriormente ${ }^{28}$. El perfil del músico popular cambió, y junto con el declinar de la industria radiofónica, Vicente Bianchi abandonó su actividad en la radio.

El compositor se adaptó a esta etapa de cambios potenciando su actividad coral, ya sea dirigiendo su propio coro como creando arreglos y composiciones para este formato. Esto coincidió con la buena recepción de sus misas ${ }^{29}$ y de su Te Deum, de modo que con un repertorio basado en arreglos de músicas tradicionales latinoamericanas, el coro de Vicente Bianchi tuvo la posibilidad de recorrer Chile y realizar presentaciones en Estados Unidos y Brasil.

25 Catálogo, O-32 y O-35 y O-36, respectivamente.

26 Bianchi afirma que Silvia Infantas debía repetir estas canciones al final de sus presentaciones en locales nocturnos. Incluso en ocasiones aquellas repeticiones habrían quedado consignadas en los contratos de trabajo por sus presentaciones en vivo.

27 Catálogo, O-111.

28 Repertorios anteriores, como bolero, tango y jazz fueron considerados como "pasados de moda" por la cultura juvenil, que prefería los nuevos estilos musicales impuestos por el movimiento de la Nueva Ola.

29 Bianchi afirma haber tenido noticias de la ejecución de sus misas en países como España, Suecia, Italia, Rusia y Suiza. Ver León 2011: 99. 


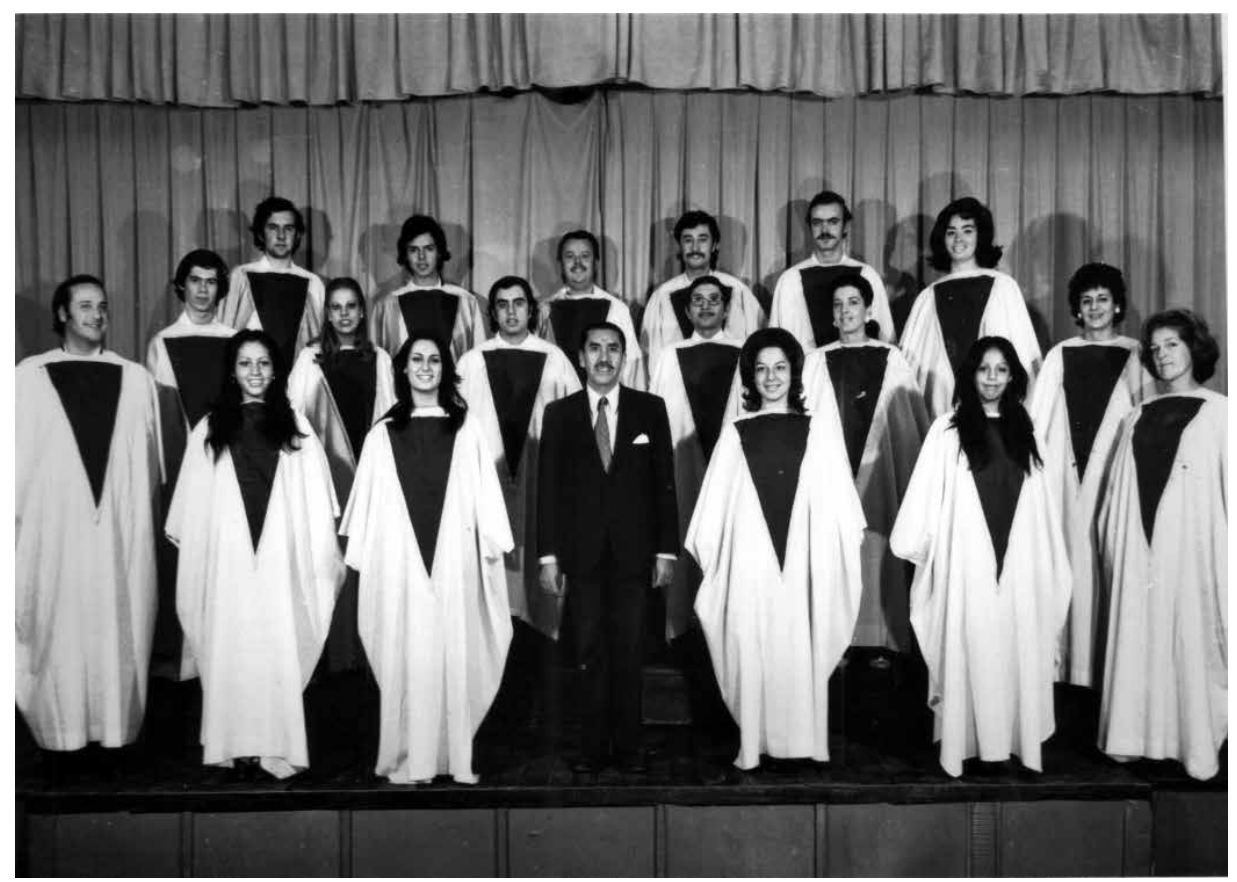

Foto 8. Coro de Vicente Bianchi. Santiago, 1974.

El mundo de la televisión en la década de 1970 no fue un espacio apto para Bianchi, de modo que se abocó a trabajar con su Coro Santa Marta, y seguir realizando arreglos de música folclórica chilena. Entre 1980 y 1985 Vicente Bianchi trabajó como director musical del Centro Cultural de La Reina, organizando los conciertos que allí se realizaban ${ }^{30}$. A fines de la década de 1980 fue director del Coro de la Universidad de Santiago.

En los últimos años Vicente Bianchi ha disminuido su actividad musical en la esfera pública, pero ha continuado escribiendo música. Ya jubilado, sigue componiendo. Parte de su tiempo lo ha dedicado a hacer arreglos orquestales de sus composiciones antiguas, para ser ejecutadas por orquestas sinfónicas de la capital (Orquesta Sinfónica de Chile, Orquesta Clásica de la Universidad de Santiago) así como también orquestas de regiones (Concepción, La Serena). También ha escrito arreglos para conjuntos de cámara, según las solicitudes que recibe para hacer el montaje de esos repertorios en formatos específicos.

En 2010 grabó un disco compacto en su casa, interpretando al piano algunos arreglos de composiciones originales y repertorios tradicionales. En 2011 el contenido de esta grabación fue editado en partitura, en una producción conjunta

30 Durante su gestión Bianchi organizó una presentación de la Orquesta Sinfónica y recitales de piano con Alfonso Montecino y Roberto Bravo. 


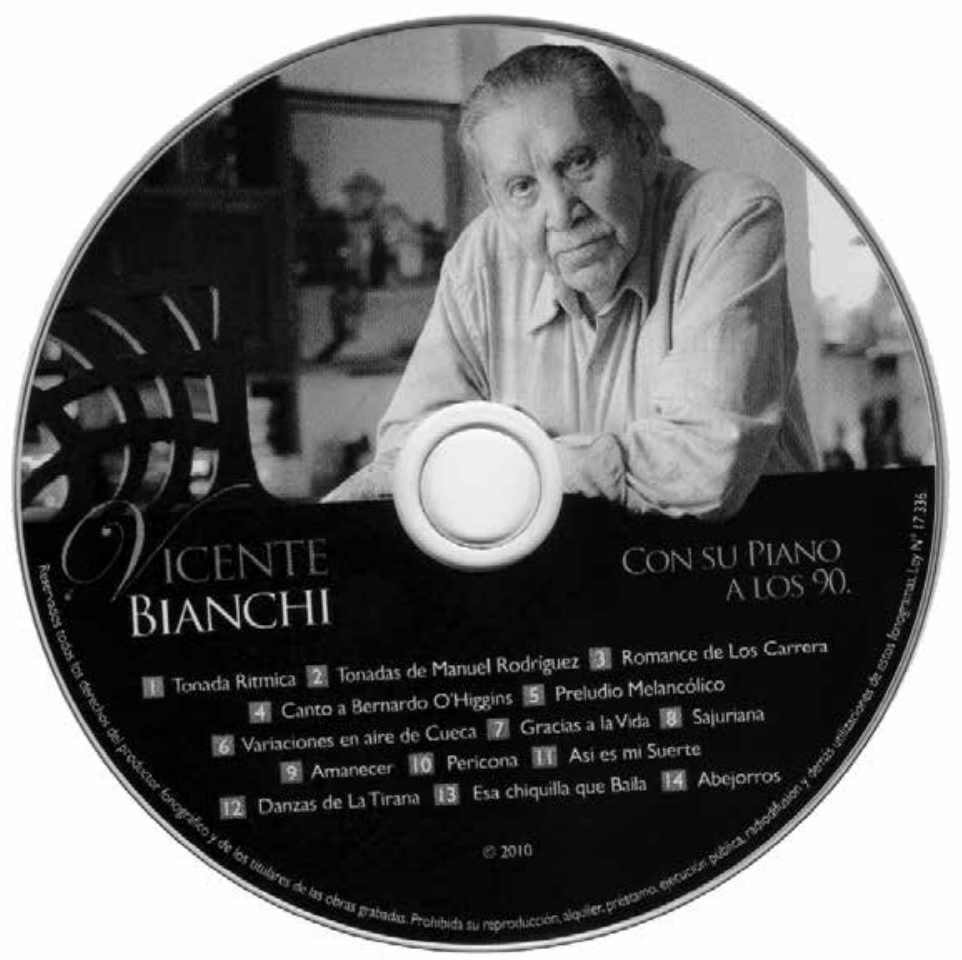

Foto 9. Imagen del CD grabado por Vicente Bianchi en 2010.

entre Escuela Moderna de Música y la Sociedad Chilena de Autores e Intérpretes Musicales, SCD.

De este modo, antes y después de haber obtenido el Premio Nacional de Artes Musicales, Vicente Bianchi ha continuado con su misma dinámica de trabajo, creando y recreando la música a la que ha dedicado su vida.

\section{VICENTE BIANCHI, PREMIO NACIONAL DE ARTES MUSICALES 2016}

El hecho que Vicente Bianchi haya sido galardonado con el Premio Nacional de Artes Musicales en Chile puede ser explicado desde varios puntos de vista. En primer lugar consideremos que dicho premio constituye el máximo galardón con que el Estado de Chile reconoce la labor de sus músicos, consistente en un monto de dinero en efectivo y una pensión vitalicia. También hay que tener en cuenta que el reglamento interno para su otorgamiento es bastante general, pues el texto de la ley establece que los premios de Artes Musicales, Audiovisuales, Artes Plásticas y Artes de la Representación serán otorgados "a la persona que se haya 
distinguido por sus logros en la respectiva área del arte 31 ". Como se aprecia, no se establece ninguna especificación respecto del perfil de los candidatos; en el caso de los músicos no se especifica si debe ser compositor o intérprete, ni tampoco el tipo de música que debiera practicar.

En segundo lugar, la ley establece que la composición del jurado que dirime esta premiación debe estar constituido por el Ministro de Educación, el Rector de la Universidad de Chile, el último galardonado con el premio en cuestión, un académico designado por el Consejo de Rectores, y un representante de la Academia Chilena de Bellas Artes.

En el caso de Vicente Bianchi el jurado que asignó el premio el 23 de agosto de 2016 estuvo constituido por la Ministra de Educación (Adriana Delpiano), el Rector de la Universidad de Chile (Ennio Vivaldi), el Rector de la Universidad de La Serena (Nibaldo Avilés, en representación del Consejo de Rectores) y el músico Fernando García (como representante de la Academia Chilena de Bellas Artes). En esta oportunidad el premiado anterior, compositor León Schidlowsky, se excusó de asistir. La circunstancia de sesionar con cuatro de los cinco miembros establecidos por ley no es extraña, pues se ha procedido de ese modo en ocasiones anteriores; por ejemplo en 2010, cuando fue premiada Carmen Luisa Letelier, también se sesionó con cuatro integrantes.

Ahora bien, ¿cuál ha sido la dinámica histórica que ha caracterizado la definición de este premio? Si revisamos la lista de galardonados desde 1945 a la fecha ${ }^{32}$, tenemos que se ha premiado a 26 músicos, en la siguiente proporción: 18 compositores de música académica, 3 intérpretes de música de concierto (Claudio Arrau, Elvira Savi y Carmen Luisa Letelier), 3 directores de orquesta (Víctor Tevah, Fernando Rosas y Juan Pablo Izquierdo), una folclorista (Margot Loyola) y un músico popular (Vicente Bianchi). (ver cuadro 1).

\section{CUADRO 1}

LISTA DE GALARDONADOS CON EL PREMIO NACIONAL DE ARTE MENCIÓN MÚSICA (1945-1986) Y PREMIO NACIONAL DE ARTES MUSICALES (1992-2016)

\begin{tabular}{|c|c|c|}
\hline $\begin{array}{c}\text { Año de } \\
\text { premiación }\end{array}$ & Nombre premiado (a) & Actividad \\
\hline 1945 & Pedro Humberto Allende & Compositor \\
\hline 1948 & Enrique Soro & Compositor y pianista \\
\hline
\end{tabular}

31 Ver art. $7^{\circ}$ de esta ley en http://premiosnacionales2016.mineduc.cl/pdf/LEY-19169_26SEP-1992.pdf

32 El Premio Nacional de Arte con menciones en Pintura o Escultura, Música y Teatro fue creado por la Ley $\mathrm{N}^{\circ} 7.638$ del 9 de noviembre de 1942 y fue sustituido por los actuales Premios Nacionales de Artes a partir de la mencionada Ley $\mathrm{N}^{\circ}$ 19.169. 


\begin{tabular}{|c|c|c|}
\hline $\begin{array}{c}\text { Año de } \\
\text { premiación }\end{array}$ & Nombre premiado (a) & Actividad \\
\hline 1951 & Domingo Santa Cruz & Compositor \\
\hline 1954 & Próspero Bisquertt & Compositor \\
\hline 1957 & Alfonso Leng & Compositor \\
\hline 1960 & Acario Cotapos & Compositor \\
\hline 1965 & Carlos Isamitt & Compositor \\
\hline 1968 & Alfonso Letelier & Compositor \\
\hline 1971 & Gustavo Becerra & Compositor \\
\hline 1976 & Jorge Urrutia Blondel & Compositor \\
\hline 1980 & Víctor Tevah & Director de orquesta \\
\hline 1983 & Claudio Arrau & Pianista \\
\hline 1986 & Federico Heinlein & Compositor \\
\hline 1992 & Juan Orrego Salas & Compositor \\
\hline 1994 & Margot Loyola & $\begin{array}{c}\text { Folclorista; investigadora e } \\
\text { intérprete }\end{array}$ \\
\hline 1996 & Carlos Botto & Compositor \\
\hline 1998 & Elvira Savi & Pianista \\
\hline 2000 & Carlos Riesco & Compositor \\
\hline 2002 & Fernando García & Compositor \\
\hline 2004 & Cirilo Vila & Compositor y pianista \\
\hline 2006 & Fernando Rosas & Director de orquesta \\
\hline 2008 & Miguel Letelier & Compositor y organista \\
\hline 2010 & Carmen Luisa Letelier & Cantante lírica \\
\hline 2012 & Juan Pablo Izquierdo & Director de orquesta \\
\hline 2014 & León Schidlowsky & Compositor \\
\hline 2016 & Vicente Bianchi & $\begin{array}{c}\text { Músico popular; pianista, } \\
\text { compositor, arreglador musical y } \\
\text { director orquestal }\end{array}$ \\
\hline
\end{tabular}


Claramente la tendencia ha sido premiar a músicos del ámbito de la música académica, formados o vinculados profesionalmente con la Universidad de Chile. Además se aprecia una notoria presencia de compositores y figuras masculinas. De este modo los grandes ausentes han sido los profesionales de la música folclórica y popular urbana (en cuanto a categorías musicales) y las mujeres (en cuanto a género).

Haber premiado en 2016 a Vicente Bianchi generó alguna crítica en el medio musical. La más recurrente fue en relación con el quiebre de la tradición en cuanto a premiar a exponentes de música académica. Si bien en primera instancia es un argumento válido desde un punto de vista estadístico, tal afirmación no se sostiene si tomamos en cuenta lo que determinan las bases de la ley que otorga el galardón. Ya se ha dicho que en ninguna parte del texto se explicita que los premiados deban pertenecer al mundo de la música de concierto. Tampoco se explicita que los premiados deban estar activos o en plena capacidad creadora, lo que ha sido un poco feliz argumento en contra del premio a Bianchi ${ }^{33}$.

Y aquí entramos en el área más compleja de este asunto: ¿Qué debemos tener en cuenta al premiar los "logros en la respectiva área del arte", en este caso de la música? En este punto algunas voces disidentes parten de la base que los logros de un músico popular no están al nivel de lo realizado por un músico que compone o ejecuta música de concierto. Este juicio de valor adolece de una importante falla conceptual, pues juzga los valores y méritos de una música X con los criterios de una música Y. Tal razonamiento tuvo su razón de ser hasta el siglo XIX, pero perdió relevancia con el desarrollo de la cultura de masas en el siglo $\mathrm{XX}$, asunto que aún no ha sido asumido por sectores importantes de la academia, ni por la crítica especializada y parte del público consumidor de música. Pensar hoy de ese modo es un acto reflejo de una élite que se resiste a perder presencia en determinados espacios y contextos, como son los conservatorios y los premios otorgados por el Estado y el resto de la institucionalidad oficial.

Entonces, ¿por qué en esta oportunidad se premió a Vicente Bianchi?

Si revisamos la estadística del Premio Nacional de Arte mención Música y del Premio Nacional de Artes Musicales podemos constatar que a lo largo de las décadas el criterio de premiación ha ido evolucionando, ampliándose más allá de la figura del compositor, es decir, del creador de música académica y de género masculino. Luego de 35 años privilegiando exclusivamente a este perfil profesional, en 1980 se premió por primera vez a un músico no compositor, a Víctor Tevah, por su aporte como director orquestal. Luego, el primer pianista clásico en ganar el premio fue Claudio Arrau en 1983, criterio refrendado con Elvira Savi en 1998.

La asignación del Premio Nacional de Artes Musicales a Margot Loyola en 1994 tuvo un mérito doble, pues fue la primera vez que se distinguió a una mujer, y al mismo tiempo a una profesional del área del folclor. Por vez primera la designación

33 Ver opinión de Juan Pablo González en http://www.elmercurio.com/blogs/2016/08/29/44618/ Premio-Nacional-de-Musica.aspx (consultado el 1/10/2017) 
del premio pasó por alto la condición de ser compositora o intérprete de música académica.

Más adelante la tendencia a premiar a músicos no compositores se manifestó en las figuras de Fernando Rosas en 2006 y de Juan Pablo Izquierdo en 2012, ambos por su labor como directores de orquesta. En 2010 Carmen Luisa Letelier fue la primera cantante lírica en recibir el galardón.

Estos hechos confirman que el criterio de premiación ha ido lentamente cambiado, lo que es muy positivo, pues instala una mirada más incluyente y desprejuiciada al momento de valorar el trabajo profesional de los músicos chilenos.

Yaquí volvemos nuevamente a la discusión respecto del valor del músico popular en comparación al músico académico, “clásico" o "docto". Quienes subvaloran el quehacer del músico popular generalmente asumen que la música popular es intrínsecamente comercial, lo que explica su masividad y arraigo en un público extenso. Asimismo, es recurrente en ellos rehusarse a poner en el mismo nivel técnico ambas músicas, pues según su argumentación una canción popular de tres minutos y una sinfonía de larga duración no son comparables a nivel de calidad y complejidad.

Entonces, ¿cuál es el valor de la música popular urbana para llegar a premiar sus logros mediante un Premio Nacional de Arte(s)? El asunto es complejo, pues la música popular no existe como un ente unitario y monolítico; existen diversas músicas populares, que también son difíciles de comparar entre sí. Porque ¿cómo comparamos a un músico de jazz como Mario Lecaros con un cantante como Germaín de la Fuente? Ambos tienen notables méritos de acuerdo con su contexto musical específico. ¿Podemos comparar el valor musical y social de un cantautor como Hugo Moraga, con otro cantautor como Ricardo Arjona? O a nivel de géneros musicales, ¿cómo comparamos el heavy metal con el bolero?

Sin pretender ser normativo en esto, sugiero valorar las diferentes músicas tomando en cuenta el mérito según la función social que cada música cumple. Ya que la música es un fenómeno multidimensional, también puede ser juzgada según esas múltiples dimensiones: algunas músicas sirven para estimular la imaginación por medio del intelecto, otras para exaltar la expresión corporal mediante el baile, otras para vehicular mensajes con contenido político, otras para expresar el amor de pareja, y así un largo etcétera. Cada una de estas funciones sociales de la música posee sus códigos específicos por los que se puede llegar a valorar cada manifestación dentro de su lógica interna; gracias a ello podemos decir que Jimi Hendrix era mejor guitarrista de blues que John Lennon. Hay que comparar lo comparable, por eso es que juzgar los méritos musicales de Bianchi en relación con Arrau es un tremendo error de concepto; es pretender comparar peras con zapallos.

A mi juicio, los logros de Vicente Bianchi que le hicieron merecedor del Premio Nacional de Artes Musicales consisten en sus diversos y excelentes aportes en diferentes áreas de la música popular urbana, aportes que le transformaron en un referente de gran importancia para quienes siguieron en esa senda:

1. Como pianista con formación en el Conservatorio Nacional de Música. Bianchi puso en práctica la experticia del pianista clásico, ampliándola más allá de la 
lectura e interpretación de obras, hacia la práctica sistemática de la improvisación en diversas músicas populares. Su labor como pianista acompañante en elencos radiales le permitió leer a primera vista, improvisar en estilo y acompañar a cantantes en cualquier tonalidad.

2. Como compositor. Su formación clásica también le permitió crear música original, tanto al piano como para agrupaciones instrumentales mayores, en el oficio cotidiano de la música popular urbana. La musicalización de versos de Pablo Neruda y sus obras de corte litúrgico generaron un importante efecto en la vida musical chilena al momento de ser estrenadas, y además han permanecido en la memoria musical del país.

3. Como arreglador musical. Su dominio de la teoría musical, la armonía y la orquestación le permitió crear sus propias versiones pianísticas y orquestales de su música original, y de infinidad de composiciones y estilos de música popular y folclórica, como bolero, tango, canción, foxtrot, tonada, cueca, pericona, vals, sajuriana, trote, samba, zamba, bambuco, vidala, guarania, triste o joropo.

4. Como director musical. Su labor profesional a lo largo de 30 años en el contexto radial y en estudios de grabación le permitieron poner en sonido una gran variedad de músicas populares en diversos formatos, desde lo puramente instrumental hasta lo coral y mixto. Gracias a la calidad de sus arreglos y dirección musical, estas músicas fueron difundidas masivamente y generaron una construcción de sentido de pertenencia en un amplio público nacional e internacional.

Parte del valor musical de Vicente Bianchi se mide también por su influencia y legado en los músicos que le siguieron: sin Bianchi no se explica la carrera musical de Valentín Trujillo, Guillermo Rifo o la Orquesta Huambaly, ni los desarrollos de movimientos musicales como el Neofolclor o la música de fusión latinoamericana.

Se puede concluir entonces que el premio a Vicente Bianchi representa un nuevo hito en la historia del Premio Nacional de Arte mención Música y de su sucesor, el Premio Nacional de Artes Musicales. Este hecho encarna el reconocimiento oficial del Estado de Chile al quehacer profesional dentro del ámbito de la música popular urbana, un área de la cultura musical del país que empieza a ser reconocida oficialmente por instituciones del Estado. Porque de eso se trata en el caso de Bianchi, de relevar su aporte y legado a la música chilena desde sus múltiples capacidades y producciones como compositor, pianista, arreglador y director musical en el ámbito de la música popular urbana.

De este modo, otorgar el Premio Nacional de Artes Musicales a Vicente Bianchi constituye un gesto altamente significativo, que abre la puerta al reconocimiento de parte del Estado a otros profesionales de la música popular, hombres y mujeres esenciales en la conformación de nuestra identidad musical. 


\section{BIBLIOGRAFÍA}

\section{LEÓN SMITH, SILVIA}

2011 Vicente Bianchi. Músico por mandato divino. Santiago: Sociedad Chilena del Derecho de Autor (SCD).

Menanteau, Álvaro (EDitor)

2011 Vicente Bianchi y su piano a los 90. Libro de partituras. Santiago: Escuela Moderna/ SCD.

Ponce, David

http://www.musicapopular.cl/artista/vicente-bianchi/ (visitado el 1/10/2017).

Entrevista a Vicente Bianchi Alarcón. Álvaro Menanteau Aravena. Santiago, 8 de septiembre de 2002. 